\title{
AtMYB14 Regulates Cold Tolerance in Arabidopsis
}

\author{
Yan Chen • Zhangliang Chen • Juqing Kang • \\ Dingming Kang • Hongya Gu • Genji Qin
}

Published online: 22 June 2012

(C) The Author(s) 2012. This article is published with open access at Springerlink.com

\begin{abstract}
Low temperature affects plant growth and crop productivity. The $C B F$ genes are a class of transcription factors that play important roles in cold response. Here we report that AtMYB14 participates in freezing tolerance in Arabidopsis by affecting expression of $C B F$ genes. The AtMYB14 gene was down-regulated by cold treatment. $A t M Y B 14$ encodes a nuclear protein that functions as an R2R3-MYB transcription activator. Knock-down of AtMYB14 by artificial microRNA increased the tolerance to freezing stress. Both the $C B F$ genes and the downstream genes were induced to a much higher level in AtMYB14 knock-down plants than in wild type under cold treatment. Our results suggest that AtMYB14 plays an important role in the plant response to cold stress.
\end{abstract}

Keywords $A t M Y B 14 \cdot$ R2R3-type MYB transcription factor $\cdot$ Cold tolerance $\cdot C B F$ genes $\cdot$ Arabidopsis

\section{Introduction}

Low temperature is one of the most stressful environmental factors affecting plant growth and development. Cold

\author{
Y. Chen $\cdot$ Z. Chen $\cdot$ D. Kang \\ College of Agronomy and Biotechnology, \\ China Agricultural University, \\ Beijing 100094, China \\ H. Gu \\ The National Plant Gene Research Center (Beijing), \\ Beijing 100101, China \\ Z. Chen $\cdot$ J. Kang $\cdot$ H. Gu $\cdot$ G. Qin $(\bowtie)$ \\ State Key Laboratory for Protein and Plant Gene Research, \\ Peking University, \\ Beijing 100871, China \\ e-mail: qingenji@pku.edu.cn
}

temperature also affects the distribution of plant species and productivity of crops (Thomashow 1998; Jiang et al. 2011; Liu et al. 2012). Plants have evolved efficient mechanisms to tolerate low temperature stress for surviving freezing periods. In the past few decades, researchers have found that many proteins are induced in the response to freezing stress. Among these, several transcriptional factors play very important roles in cold tolerance. The $\mathrm{C}$-repeat binding factor (CBF) proteins, including CBF1 (DREB1b), CBF2 (DREB1c), and CBF3 (DREB1a), are pivotal transcriptional factors in this process (Stockinger et al. 1997; Liu et al. 1998). After induction by low temperature, CBF proteins regulate the expression of numerous cold responsive (COR) genes and increase the freezing tolerance of plants by binding to C-repeat/dehydration-responsive (CRT/DRE) elements (A/GCCGAC) in the promoter regions of these genes (Baker et al. 1994; Yamaguchi-Shinozaki and Shinozaki 1994; Thomashow 1999). The three CBF genes, which all belong to the AP2/EREBP gene family (Stockinger et al. 1997; Liu et al. 1998), play different roles in the cold response pathway, although they are all induced by cold stress (Novillo et al. 2004, 2007), suggesting a complex cold response signaling network. Downstream genes in the cold stress response include KIN1 (Kurkela and Franck 1990), COR47 (Gilmour et al. 1992), COR15A (Lin and Thomashow 1992), and RD29A (Nordin et al. 1993), which are all COR genes. These genes are activated by CBF transcription factors in diverse plant species (Liu et al. 2012; Wang et al. 2011; Zhang et al. 2011).

$C B F$ genes are tightly regulated. The expression of $C B F 3$ is activated by a transcription factor called ICE1 (inducer of $\mathrm{CBF}$ expression 1). In contrast to $C B F 3$, which is induced in cold stress, ICE1 is expressed constitutively in Arabidopsis (Chinnusamy et al. 2003). ICE1 is a MYC-like transcription factor that contains a bHLH domain. ICE1 binds to the 
MYC recognition elements CANNTG in the promoter region of $C B F 3$ (Chinnusamy et al. 2003). Overexpression of $I C E 1$ causes elevated expression of $C B F 3, C B F 2$ and downstream genes in cold stress, and thus enhances freezing tolerance to low temperature. Other proteins regulate the expression of $C B F$ genes through interaction with ICE1. HOS1 (high expression of osmotically responsive gene 1) - a RING E3 ligase - interacts with ICE1 and mediates the ubiquitination and degradation of ICE1, which negatively regulates $C B F$ genes (Lee et al. 2001; Dong et al. 2006). Genetic analysis shows that the transgenic plants in which HOS1 is over-expressed are hypersensitive to cold stress (Dong et al. 2006), whereas hos1 mutants show enhanced expressions of $C B F$ genes and their target genes in cold stress (Lee et al. 2001). SIZ1 (SAP and Miz1) - a SUMO (small ubiquitin-related modifier) E3 ligase-regulates ICE1 positively by adding SUMO to K393 of ICE1 (Gilmour et al. 1992). The sizl mutant shows reduced expressions of $C B F$ genes and their target genes, and thus is sensitive to freezing. A MYB transcription factor AtMYB15 regulates $C B F$ genes negatively. AtMYB15 lossof-function mutants increase tolerance to cold stress through elevating the expression of $C B F$ genes. AtMYB15, interacting with ICE1, binds to MYB recognition sequences in the promoter regions of $C B F$ genes and down-regulates $C B F$ genes (Agarwal et al. 2006).

In recent years, MYB transcription factors have been found to regulate many physiological and biochemical processes, such as secondary metabolism, plant development and stress response (Kranz et al. 1998; Stracke et al. 2001; Chen et al. 2006a). MYB proteins belong to the largest family of transcription factors in Arabidopsis (Riechmann and Ratcliffe 2000). The DNA-binding domains of MYB proteins contain one to three imperfect repeats, named R1, R2 and R3, respectively. These repeats form a helix-turnhelix structure comprising 51-53 amino acids (Stracke et al. 2001). Among 198 MYB genes in Arabidopsis, 126 encode R2R3-MYB transcription factors, 5 encode R1R2R3-MYB proteins, 64 encode other MYB-related proteins, and 3 encode atypical MYB proteins (Chen et al. 2006a).

Many genes participate in the complex network of plant cold responses; however, the mechanisms by which plants respond to cold stress remain elusive. We previously found that many MYB genes responded to stress treatments (Chen et al. 2006a). In this study, we identified a MYB transcription factor, AtMYB14, as being involved in cold tolerance in Arabidopsis. Knock-down of AtMYB14 by artificial microRNA led to increased tolerance to low temperature. The expression of $C B F$ genes and cold-response downstream genes were increased to a much higher degree in AtMYB14 knock-down plants than in wild type under cold treatment. Our results indicate that AtMYB14 is an important element in the signaling pathway of cold tolerance.

\section{Materials and Methods}

Plant Materials and Growth Conditions

Arabidopsis thaliana ecotype Columbia-0 (col-0) was used. Plants were grown as described previously (Qin et al. 2005). Briefly, seeds were plated on $1 / 2 \times$ MS medium (half strength Murashige and Skoog medium) containing $1 \%$ sucrose. The plates were kept at $4{ }^{\circ} \mathrm{C}$ in the dark for 3 days for synchronization. The plates were transferred to a chamber at $22{ }^{\circ} \mathrm{C} \pm 2{ }^{\circ} \mathrm{C}$ under long-day conditions (16 h light $/$ $8 \mathrm{~h}$ dark). After 7 days the seedlings were transferred to soil and placed in a greenhouse at $22{ }^{\circ} \mathrm{C} \pm 2{ }^{\circ} \mathrm{C}$ under long-day conditions ( $16 \mathrm{~h}$ light $/ 8 \mathrm{~h}$ dark).

\section{Cold Treatment and Freezing Tolerance Test}

For cold treatment in experiments to determine AtMYB14 regulation by cold stress, 2-week-old wild-type plants on $1 / 2 \times$ MS medium were placed in a chamber at $0{ }^{\circ} \mathrm{C}$ under continuous light for $0,0.5,1,3,6,12$, or $24 \mathrm{~h}$.

The freezing tolerance test of Arabidopsis was described previously with some modifications (Zhu et al. 2004). Three-week-old plants in soil in the greenhouse were placed in a freezing chamber at $-1{ }^{\circ} \mathrm{C}$. The chamber cooled at a rate of $-1{ }^{\circ} \mathrm{C} / \mathrm{h}$, then held at $-8^{\circ} \mathrm{C}$. After exposure to $-8{ }^{\circ} \mathrm{C}$ for $2 \mathrm{~h}$, plants were transferred to a greenhouse at $22^{\circ} \mathrm{C}$ under long-day conditions. The test was conducted in the dark in a freezing chamber. Survival rates of plants were assessed after 7 days.

RNA extraction, Reverse Transcription and Real-Time Quantitative PCR

Total RNA was extracted from 2-week-old Arabidopsis seedlings using Trizol reagent. The total RNA was treated with DNase I (TaKaRa, Tokyo, Japan) and then reversetranscribed according to the producer's manual and as described previously (Liu et al. 2008). The real-time quantitative PCR conditions were as described by Xing et al. (2007). Briefly, the reaction was performed on an Option 2 Continuous Fluorescence Detector (MJ Research, Waltham, MA) and a SYBR Green realtime PCR Master Mix (TOYOBO, Tokyo, Japan) was used. The real-time PCR products were assessed by melting curve and gel electrophoresis to ensure the specificity of the reaction. Three technical replicates were carried out for each biological sample. Cycling conditions were $95{ }^{\circ} \mathrm{C}$ for $2 \mathrm{~min}, 95^{\circ} \mathrm{C}$ for $20 \mathrm{~s}, 55^{\circ} \mathrm{C}$ for $20 \mathrm{~s}$, and $72{ }^{\circ} \mathrm{C}$ for $20 \mathrm{~s}$. The relative expression level of each gene was calculated using the $2^{-\Delta \Delta \mathrm{CT}}$ method. The Arabidopsis elongation factor gene $E F 1-1$ was used as an internal control. The gene-specific primers for EF1-1 (AT1G07930) were (5'-CCT CCC AGG CTG ATT GTG CT-3' and 5'-TAT 
TTG GGG GTG GTG GCA TC-3'). The other gene-specific primers are as follows: AtMYB14 (5'-GTT AAC GGA ATT AAC GAG ACC ACA A-3' and 5'-AAAC TAT CAT CTA TCA AGG CAG AAA-3'), CBFl (5'-CTG AAG TGA GAG AGC CAA AC-3' and 5'-AGT CAG CGA AGT TGA GAC AT-3'), CBF2 (5'-CTA TTT ATA CGC CGG AAC AG-3' and 5'-GCC ATG TTA TCC AAC AAA CT-3'), CBF3 (5'-TTT CAG GAT GAG ATG TGT GA-3' and 5'-CTT CTG CCA TAT TAG CCA AC-3'), RD29A (5'ATC ACT TGG CTC CAC TGT TGT TC-3' and 5'-ACA AAA CAC ACA TAA ACA TCC AAA GT-3'), KIN1 (5'ACC AAC AAG AAT GCC TTC CA-3' and 5'-CCG CAT CCG ATA CAC TCT TT-3'), COR15A (5'-GGC CAC AAA GAA AGC TTC AG-3' and 5'-CTT GTT TGC GGC TTC TTT TC-3'), COR47 (5'-AAG CTT CAC CGA TCC AAC AG-3' and 5'-TAC CGG GAT GGT AGT GGA AA-3').

\section{Subcellular Localization}

For subcellular localization, the full-length cDNA of AtMYB14 was amplified using the primers AtMYB14-F (5'GAA GAT CTA TGG GAA GAG CAC C-3') and AtMYB14R (5'-GCT CTA GAT TAA AAC TCG GGT A-3'). The PCR products were digested with $B g l \mathrm{II}$ and $X b a \mathrm{I}$, and then cloned into the vector pRTL-GFP (Yi et al. 2002). The construct was bombarded into epidermal cells of onions as described previously (Li et al. 2006a).

\section{Transactivation Activity Assays}

The transcription activity of AtMYB14 was examined by a yeast one-hybrid assay using the yeast strain EGY48. We evaluated AtMYB14 activity using deletion mutants: the N-terminal region of 1-115 amino acids containing the MYB domain, a short (25 aa) region corresponding to amino acids 224-249 containing the conserved M(E/D) FWFD motif in the C-terminal region, the C-terminal region of amino acids 116-223 without the short 25 aa region, and the $\mathrm{C}$-terminal region of amino acids 116249. The deletions of AtMYB14 were amplified using the following primers: AtMYB14-1/115 (5'-GGA ATT CAT GGG AAG AGC AC-3' and 5'-CCG CTC GAG TCT TTT CTT CA-3'), AtMYB14-224/249 (5'-CCG GAA TTC ATG AAT GAT GAC ATG GA-3', AtMYB14-116/ 223 (5'-GGA ATT CAT GCT CAG CAA AA-3' and 5'-CCG CTC GAG ATA CAA CTT AG-3'), and 5'-CCG CTC GAG TTA AAA CTC GG-3'), AtMYB14-116/249 (5'-GGA ATT CAT GCT CAG CAA AAA TCT AAA C$3^{\prime}$ and 5'-CCG CCG CTC GAG TTA AAA CTC GGG TAT G-3'). The products were digested with EcoR I and Xho I and cloned into the vector of pYF503 (Ye et al. 2004). Transactivation activity assays were performed as described previously (Li et al. 2006b).
Construction of $\operatorname{PrO}_{A t M Y B} 14::$ GUS and Histochemical GUS Assays

For detection of AtMYB14 promoter activity, the promoter was amplified and cloned in front of a $\beta$-glucuronidase (GUS) reporter gene as follows. The primers AtMYB14pro-F (5'-GGA TCC TGA TGG AAT TAG ATA TCG ACG-3') and AtMYB 14-pro-R (5'-ACT AGT GAG AGC TCG CCA ATT AGA ATG-3'), were used for amplification. The PCR products were digested with BamH I and Spe I, and then cloned into the vector pCAMBIA1381Xa to generate Pro $_{\text {AtMYB } 14:}:$ GUS. GUS activity was analyzed as described previously (Chen et al. 2006b) using 2-, 4-, 8-, and 16-day-old seedlings. Briefly, whole seedlings were infiltrated in GUS staining solutions containing $0.5 \mathrm{mg} / \mathrm{ml}$ 5-bromo-4-chloro-3-indolyl glucuronide in $0.1 \mathrm{M} \mathrm{Na}_{2} \mathrm{HPO}_{4}$, pH 7.0, $10 \mathrm{mM} \mathrm{Na}{ }_{2}$ EDTA, $0.5 \mathrm{mM}$ potassium ferricyanide/ferrocyanide, and $0.06 \%$ Triton $\mathrm{X}-100$ and incubated at $37{ }^{\circ} \mathrm{C}$ overnight. The stained plants were then cleared in $70 \%$ ethanol.

AtMYB14 Overexpression and Artificial microRNA of AtMYB 14

To generate the AtMYB14 overexpression construct, the fulllength cDNA of AtMYB14 was amplified using primers AtMYB14-OX-F (5'-CTC GAG AAA AAG AAT GGG AAG AGC ACC-3') and AtMYB14-OX-R (5'-TCT AGA AAA TCA AAA TTA AAA CTC GGG-3'). The amplified fragments were digested by Xho I and $X b a \mathrm{I}$, and then cloned into the vector pJim19, in which AtMYB14 is driven by the CaMV35S promoter. Transformation mediated by Agrobacterium tumefaciens strain GV3101 into Arabidopsis plants was carried out by the floral dipping method as described previously (Qin et al. 2005).

The engineering of artificial microRNA was described by Schwab et al. (2006). For construction of artificial microRNA AtMYB14, the Web MicroRNA Designer (http:// www.weigelworld.org) was used to identify the sequences. The primers used were as follows: PBS300-A (5'-CTG CAA GGC GAT TAA GTT GGG TAA C-3'), PBS300-B (5'-GCG GAT AAC AAT TTC ACA CAG GAA ACA G-3'), I miR-s (5'-gaT AA TTA TCT ACC GAT ATG GCG tct ctc tttt gta ttc c-3'), II miR-a (5'-gaC GCC ATA TCG GTA GAT AAT Tat caa aga gaa tca atg a-3'), III miR-s (5'gaC ACC ATA TCG GTT GAT AAT Tat cac agg tcg tga tat g-3'), IV miR-a (5'-gaT AAT TAT CAA CCG ATA TGG TGt cta cat ata tat tcc t-3'). The amiRNA precursor was cloned into $\mathrm{pBS}$ and confirmed by sequencing. The pBSamiRNA14 was digested with Spe I and Xho I, and the target DNA fragment was cloned into pJim19 to generate the construct pJim19-amiRNA14, which was then transformed into Arabidopsis plants by floral dipping (Qin et al. 2005). 
T2 seeds from AtMYB14 overexpression lines and amiRNA14 T1 transgenic lines were plated on 1/2MS medium containing $50 \mu \mathrm{g} / \mathrm{ml}$ kanamycin. The numbers of green and yellow seedlings were counted. If the ratio of green to yellow seedlings from $\mathrm{T} 1$ progenies was $3: 1$, the $\mathrm{T} 1$ line was selected as a transgenic line with a single copy of the T-DNA. The green plants were then transferred to soil. The T3 seeds were again plated on $1 / 2 \mathrm{MS}$ medium containing $50 \mu \mathrm{g} / \mathrm{ml}$ kanamycin. Lines with no segregation were selected as AtMYB14 overexpression or amiRNA14 homozygous lines.

\section{Results}

Expression of AtMYB14 is Down-Regulated by Cold Stress

We have previously identified 163 MYB genes and found that many of them are regulated by stress treatments (Chen et al. 2006a). In order to search for possible functions of MYB transcription factors in cold tolerance, we first examined changes in their expression under cold treatment using real-time quantitative PCR. The results showed that one transcription factor, AtMYB 14, was significantly downregulated by cold stress, whereas the relative expression level of $A t M Y B 14$ at normal temperature $\left(22^{\circ} \mathrm{C}\right)$ at different times showed no changes in expression (data not shown), indicating that changes in expression of AtMYB14 were not due to circadian rhythm. As shown in Fig. 1, AtMYB14 transcripts decreased rapidly during $0{ }^{\circ} \mathrm{C}$ treatment. The level of gene expression was reduced by more than half after cold treatment for $30 \mathrm{~min}$. After $6 \mathrm{~h}$ at $0{ }^{\circ} \mathrm{C}$, expression of AtMYB14 reached its lowest level (Fig. 1a). To further confirm the down-regulation of AtMYB14 by cold stress, we cloned the promoter region of $A t M Y B 14$ and fused it to the uidA gene to generate the Pro $_{A t M Y B 14}::$ GUS construct. Pro $A t$ MYB 14: :GUS transgenic plants were treated at $0{ }^{\circ} \mathrm{C}$ for $24 \mathrm{~h}$; GUS staining showed that the GUS activity decreased significantly under cold treatment in the seedling stage (Fig. 1b). Taken together, these results indicate that AtMYB14 is down-regulated by low temperature.

\section{AtMYB14 Encodes an R2R3-MYB Domain Protein}

Bioinformatics analysis revealed that AtMYB14 encodes a protein of 249 amino acids that contains an R2R3-MYB domain (Fig. 2a). The MYB domain is a DNA binding domain that consists of one to three imperfect repeats named R1, R2 and R3 (Chen et al. 2006a). Within the large MYB protein family in Arabidopsis, AtMYB14 protein sequence shares the highest similarity with AtMYB15 in the MYB repeats. Their MYB domains share $82.5 \%$ amino acid sequence identity (Fig. 2a). Interestingly, AtMYB14 contains one $\mathrm{C}$-terminal motif M (E/D) FWFD similar to that of
AtMYB15 involved in cold tolerance (Fig. 2a) (Agarwal et al. 2006). According to sequence similarity, AtMYB14 is grouped with AtMYB15 in the phylogenetic tree (Fig. 2b).

AtMYB14 is a Nuclear Protein and has Transcriptional Activation Activity

In order to test the subcellular localization of the AtMYB14 protein, an AtMYB14-GFP construct was generated by fusing full-length AtMYB14 cDNA in frame with the Nterminus of the green fluorescent protein $(G F P)$ gene. The fusion protein was driven by a CaMV $35 \mathrm{~S}$ promoter in the AtMYB14-GFP construct. This construct was bombarded into onion epidermal cells. The control construct in which the GFP gene was driven by the CaMV $35 \mathrm{~S}$ promoter was also transformed by bombardment. In AtMYB14-GFP transgenic cells, strong GFP fluorescence was found in the nucleus, and a weak fluorescence outside the nucleus was also observed (Fig. 3a), whereas the GFP control was localized throughout the cell (Fig. 3a). These results indicate that AtMYB14 is a nuclear protein.

To examine the transcription activity of AtMYB14, we used a yeast one-hybrid system (Ye et al. 2004), in which full-length AtMYB14 and a series of deletions were fused to the GAL4 DNA-binding domain and then cotransformed with the $L a c Z$ reporter into yeast cells. The results showed that the full-length protein displayed a high transactivation activity (Fig. 3b). This suggests that AtMYB14 might be a transcriptional activator. No GUS activity could be detected for the $\mathrm{N}$-terminal region of $1-$ 115 amino acids containing the MYB domain (similar activity to the negative control; Fig. $3 \mathrm{~b}$ ). The C-terminal region of 116-249 amino acids showed high GUS activity, suggesting that this region may contain the activation domain. Interestingly, the short region comprising amino acids 224-249 containing the conserved C-terminal M(E/D)FWFD motif showed GUS activity. The Cterminal region of amino acids 116-223 without this short region also had transactivation activity (Fig. 3b), which differs from that observed in AtMYB15 (Chen et al. 2006b). The results suggest that AtMYB14 may be a transcriptional activator and that amino acids 116-249 in the $\mathrm{C}$-terminal region are responsible for the transactivation activity.

\section{Expression Pattern of AtMYB14}

To elucidate the expression pattern of AtMYB14, we first analyzed its transcript level in different tissues using realtime quantitative PCR in Arabidopsis. AtMYB14 was expressed in imbibed seeds, 5-day-old roots, the shoots of 2-week-old seedlings, siliques and flowers, with the highest level in the roots (Fig. 4). Strong GUS activity was detected 
Fig. 1 Expression of AtMYB14 is down-regulated by cold treatment. a AtMYB14 transcript levels of 2-week-old wild type plants under $0{ }^{\circ} \mathrm{C}$ treatment were revealed by real-time quantitative PCR analysis. EF11 was used as an internal control. Error bars SD of biological replicates $(n=3)$. b GUS staining of Pro $_{A t M Y B 14}: \because$ GUS transgenic plants with or without $0{ }^{\circ} \mathrm{C}$ treatment for $24 \mathrm{~h}$. The transgenic plants were treated at $4{ }^{\circ} \mathrm{C}$ or $22^{\circ} \mathrm{C}$ at the end of 2,4 , 8 and 16 days after germination. Bars $100 \mu \mathrm{m}$ in 2- and 4-dayold plants, $400 \mu \mathrm{m}$ in 8-day-old plants, and $800 \mu \mathrm{m}$ in 16-dayold plants a

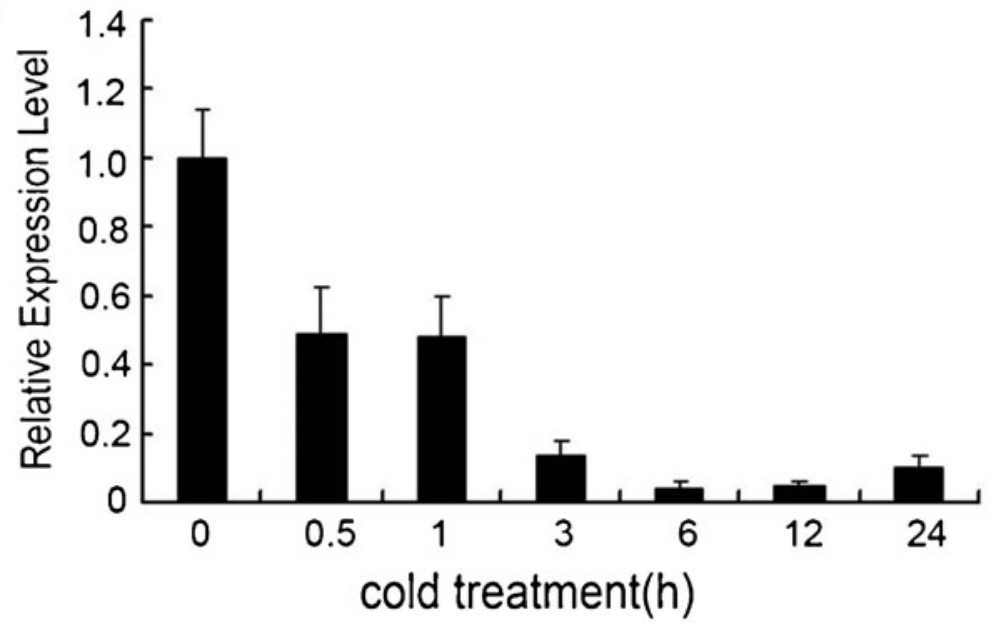

b
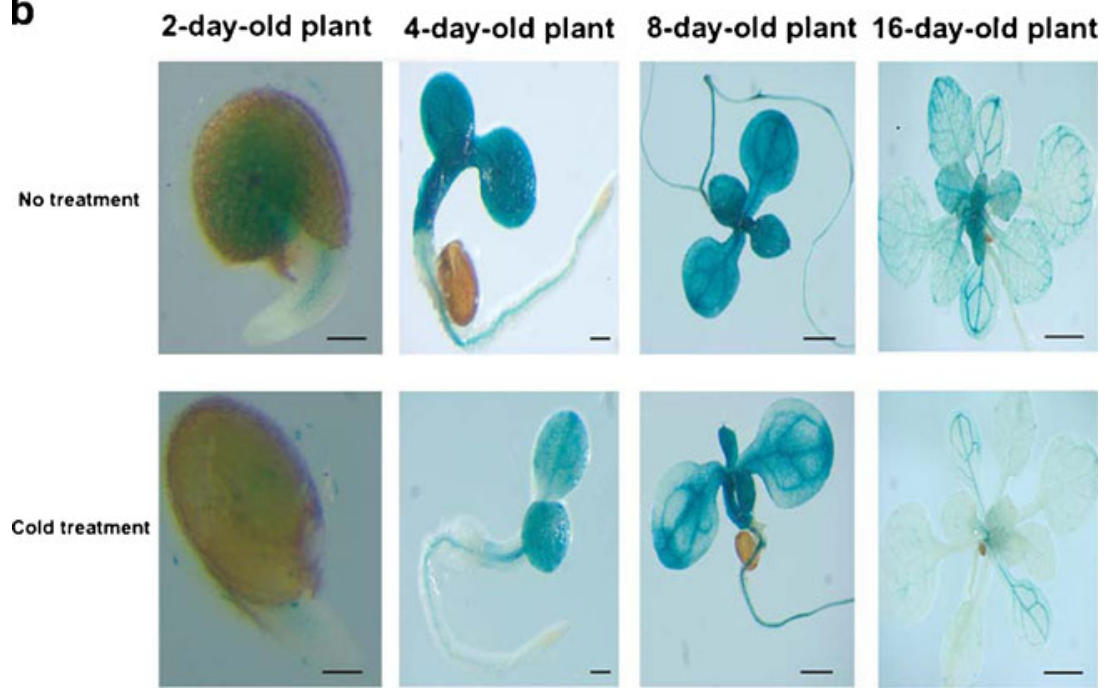

in hypocotyls, cotyledons and roots in 4-day-old Pro AtMYв 14::GUS transgenic seedlings and 8-day-old Pro ${ }_{A t-}$ MYB 14::GUS transgenic seedlings (Fig. 1b), which was consistent with the real-time PCR results. GUS staining in 16-day-old seedlings was faded in the roots and old leaves when compared with that observed in 8-day-old seedlings, indicating that AtMYB14 may be developmentally regulated.

\section{Knock-down of AtMYB14 Increases Tolerance to Cold} Stress

To investigate the function of AtMYB14 in cold stress, we knocked down the transcript level of AtMYB14 in transgenic plants using artificial miRNA technique. Six transgenic plants were obtained and the transcript levels of AtMYB14 were analyzed using real-time quantitative PCR. Two independent lines - amiRNA14-2 and amiRNA14-10 - showed a significant reduction in AtMYB14 transcript levels (Fig. 5a). The expression level of $A t M Y B 15$, which has high similarity with $A t M Y B 14$, was not affected by the artificial microRNA in the
amiRNA14-2 line (data not shown). T3 homozygous lines of amiRNA14-2 were selected for tolerance analysis in cold stress because of their lower AtMYB14 transcript levels (Fig. 5a). We also generated a construct in which AtMYB14 was driven by the CaMV $35 \mathrm{~S}$ promoter. We obtained 11 transgenic plants and transcript levels of AtMYB14 were analyzed. Two homozygous transgenic plants with increased AtMYB14 expression were selected and named as OX14-9 and OX14-15 (Fig. 5b). The T3 homozygous lines of OX149 was chosen for cold tolerance analysis because of its higher AtMYB1 expression level. As shown in Fig. 5c,d, following treatment of these plants at $-8{ }^{\circ} \mathrm{C}$ for $2 \mathrm{~h}$, the survival rate of amiRNA14-2 was $87 \%$, whereas the survival rate of the wild type control was only $71 \%$. The survival rate of OX14-9 lines was $67 \%$, which was not significantly different from that of the wild type control. This indicated that the amiRNA14-2 plants showed enhanced tolerance, but OX14-9 plants had no obvious difference from wild-type plants in cold tolerance. These results indicate that AtMYB14 may be involved in signal transduction in the plant cold response. 
Fig. 2 Sequence analysis of AtMYB14. a Amino acid alignment of AtMYB14 and AtMYB15. Black lines R2 and R3 MYB domains. Identical amino acid residues are shown in the same color. The conserved motif M(E/D)FWFD in the C-terminal is underlined by a thick red line. b Phylogenetic tree of AtMYB14 and some other R2R3-MYB proteins from Arabidopsis. The phylogenetic tree was constructed on the basis of Nterminal domains of R2R3MYB using the CLC sequence viewer program (http:// www.clcbio.com). AtMYB14 and AtMYB15 are grouped together a

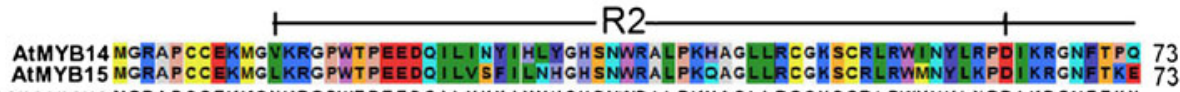
consensuS MGRAPCCEKMGXKRGPWTPEEDQI I XXX IXXXGHSNWRALPKXAGLLRCGKSCRLRWXNYLXPDIKRGNFTXX Ш

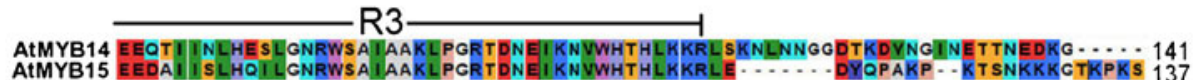
ATMYB15 EEDAI I SLHQILGNRWSAI AAKLPGRTDNEI KN WWHTHLKKRLE........ DYOPAKP. . KT SNKKKGTKPKS 137 consensus EEXXI I XLHXXLGNRWSA IAAKLPGRTDNE I KN VWHTHLKKRLXKNLNNGGDXXXXXXINXTXNXXKGTKPKS

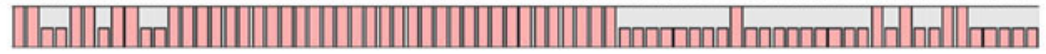

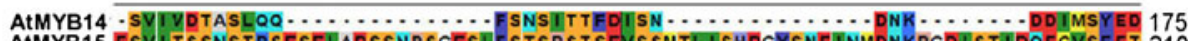
ATMYB15 ESVIITS SNSTRSESELADSSNPSGESLLSTSPST SEVS SMTLI SHDG Y SNEINMDNKPGDIISTIDOECVSFEET 210 consenSUS ESVI $X X X X S X X X E S E L A D S S N P$ SGES LF $X X S X X T \times X X S X N T L$ I SHDGYSNE INMDNKPGO I ST IDXXXXSXEX

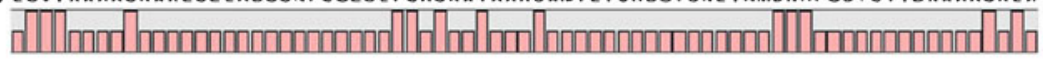

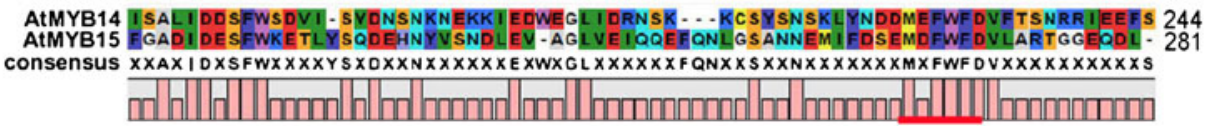

AtMYB14 DIPEF 249

AtMYB15 - LAGL 285

consensus $\underline{0 \times x \times x}$

핌ำ

b

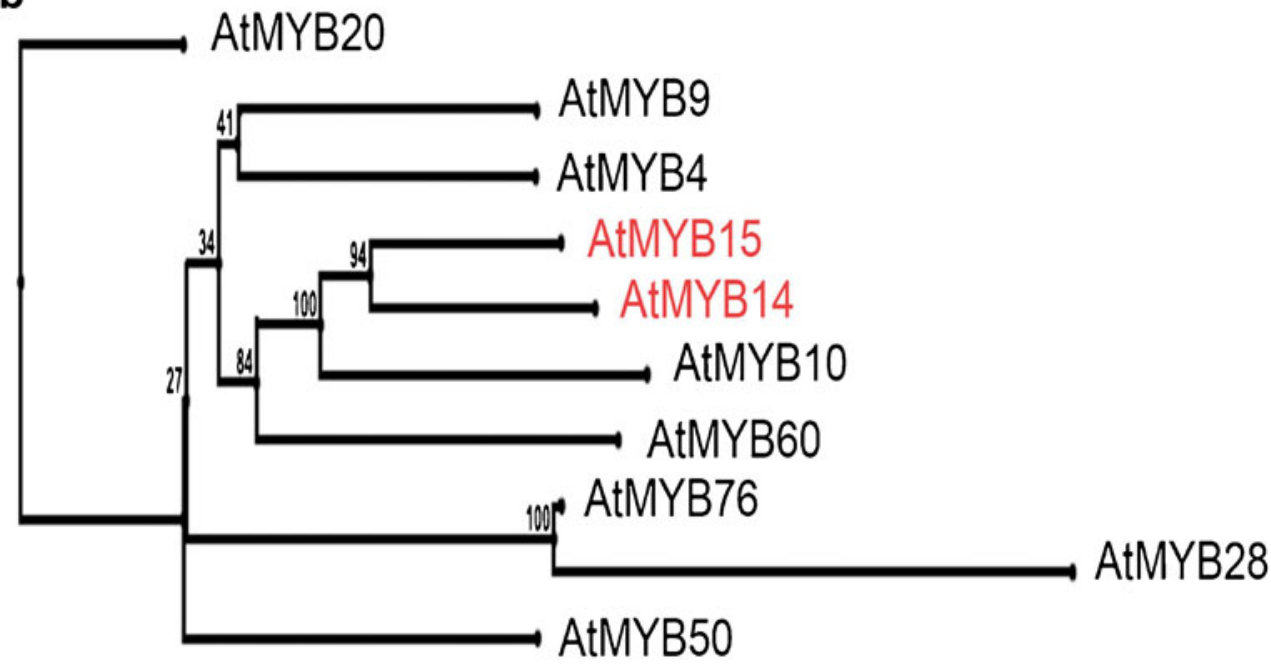

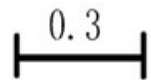

Knockdown of AtMYB14 Increases Expression of $C B F$ Genes Under Cold Treatment

To determine the molecular mechanisms by which AtMYB14 regulates cold tolerance, we examined changes in expression of $C B F$ genes, which are known regulators of cold response. When amiRNA14-2, OX14-9 and wild-type plants were treated at $0{ }^{\circ} \mathrm{C}$ for $24 \mathrm{~h}$, the expression levels of all three $C B F(C B F 1, C B F 2$, and $C B F 3)$ were increased much more in amiRNA14-2 than in wild-type or OX14-9 lines (Fig. 6a-c). The expression levels of these three genes in OX14-9 lines were a little lower than those in wild-type plants (Fig. 6a-c). These results suggest that AtMYB14 may affect upstream genes in the cold signaling pathway. We next analyzed changes in the expression of $C B F$ downstream genes. Consistent with the changes in $C B F$ genes, the expression levels of $C B F$ downstream genes, including KIN1, COR15A, COR47, and RD29A in amiRNA14-2 lines were elevated to levels higher than those in wild-type or OX14-9 lines when they were treated at $0{ }^{\circ} \mathrm{C}$ for $24 \mathrm{~h}$ (Fig. 6d-g). These results suggest that the increased freezing tolerance in amiRNA14-2 plants resulted from the much higher expression of $C B F$ genes and their downstream genes under cold treatment. 
Fig. 3 Subcellular localization and transactivation activity of AtMYB14. a Subcellular localization of AtMYB14. Images taken using a fluorescence microscope (left), bright microscope (middle) were merged (right). Bar $50 \mu \mathrm{m}$. b Transactivation of AtMYB14 in yeast. Full-length and deletion mutants of AtMYB 14 cDNA are illustrated on the left and transactivation activity on the right. Empty pYF503 was used as the negative control and pYF504 used as the positive control

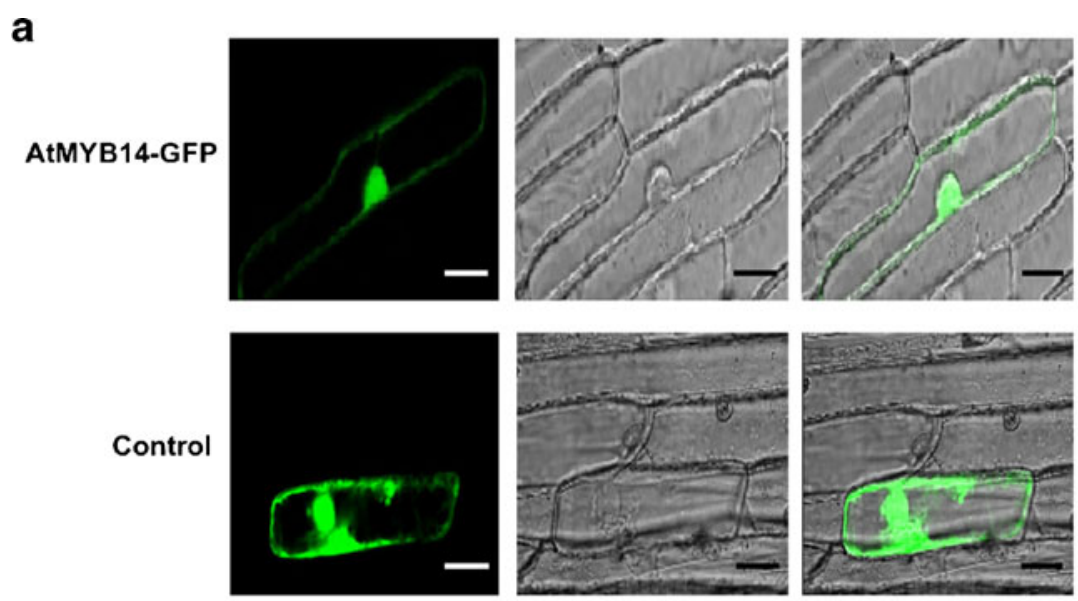

b

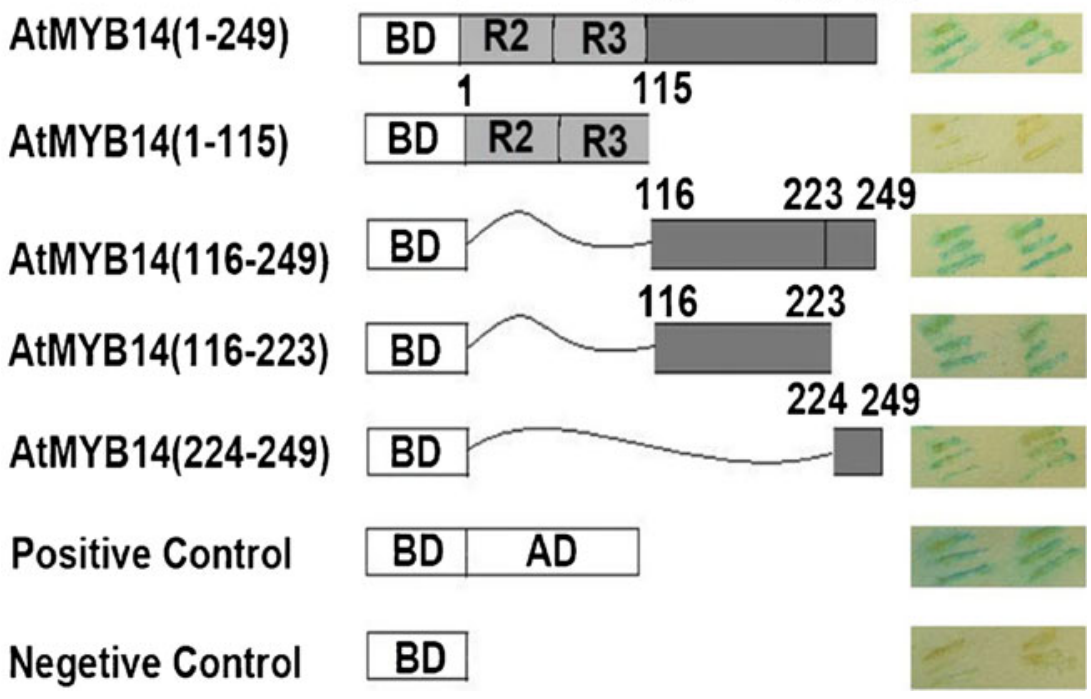

\section{Discussion}

Plants survive periods of low temperature in their life cycles by changing gene expression profiles to make physiological adjustments. Some important transcription factors and many

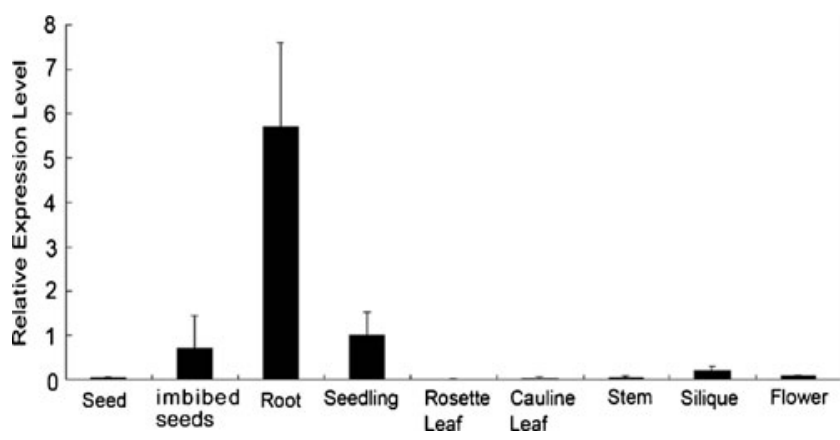

Fig. 4 Expression pattern of AtMYB14. Analysis of relative AtMYB14 transcript levels in different tissues by real-time quantitative PCR. EF1-1 was used as an internal control. The expression level of AtMYB14 in seedlings was set to 1.0. Error bars SD of biological replicates $(n=3)$
COR genes are induced in cold stress to realize these physiological alterations (Thomashow 1999; Viswanathan and Zhu 20021; Xiong et al. 2002). $C B F$ genes are induced by exposure to low temperature and encode pivotal transcription factors that activate the downstream COR genes in the cold signaling pathway (Stockinger et al. 1997; Gilmour et al. 1998). In our study, we found that AtMYB14, an R2R3-type transcription factor with transactivation activity, participated in cold tolerance by affecting $C B F$ genes. The expression of AtMYB14 was down-regulated by cold treatment. Knock-down of AtMYB14 by artificial microRNA in transgenic plants caused higher resistance to cold stress through regulating $C B F$ genes. We suggest that AtMYB14 plays an important role in cold response in Arabidopsis.

AtMYB14 was found to have a high similarity with AtMYB15 in MYB domains. AtMYB15 was found to bind to the promoters of $C B F$ genes and functioned as a negative regulator of their expression (Agarwal et al. 2006). Although both MYB proteins played a negative role in cold response, there were differences. First, AtMYB15 is up- 
Fig. 5 Increased freezing tolerance of the AtMYB14 knock-down lines. a Real-time quantitative PCR analysis of relative AtMYB14 transcript levels in amiRNA14 lines; the expression level of AtMYB14 in wild type plants was set to 1.0 . b Real-time quantitative PCR analysis of relative AtMYB14 transcript levels in OX14 lines; the expression level of AtMYB14 in wild type plants was set to 1.0. c Freezing test carried out using wild type, OX14-9 and amiRNA14-2 plants. All plants were grown in soil at $22{ }^{\circ} \mathrm{C}$ for 3 weeks and then transferred into a freezing chamber at $-1{ }^{\circ} \mathrm{C}$. The chamber cooled at the rate of $-1{ }^{\circ} \mathrm{C} / \mathrm{h}$, then held at $-8{ }^{\circ} \mathrm{C}$. After exposed to $-8{ }^{\circ} \mathrm{C}$ for $2 \mathrm{~h}$, plants were transferred to a greenhouse at $22{ }^{\circ} \mathrm{C}$. After 7 days, the plants were photographed. d Survival rates of amiRNA142 and OX14-9 were analyzed on the 7 th day after the freezing test. Error bars SD of biological replicates $(n=3)$ a

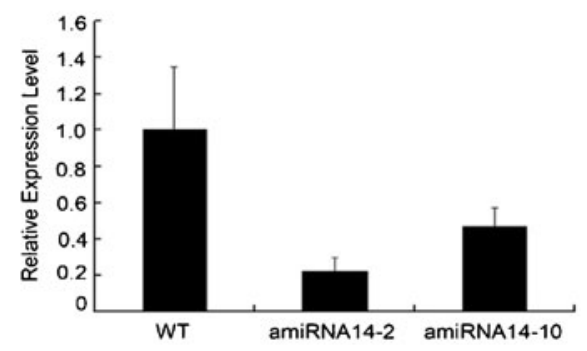

b

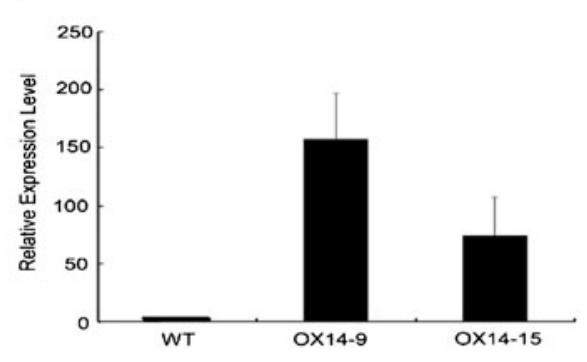

C WT
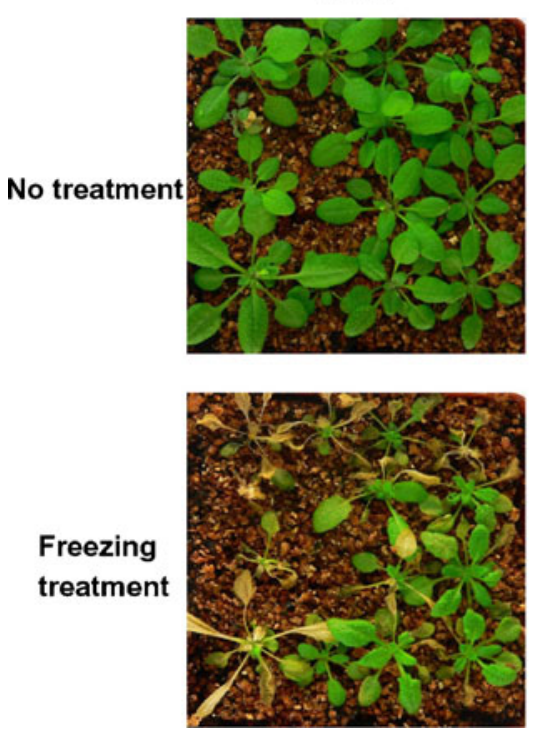

d

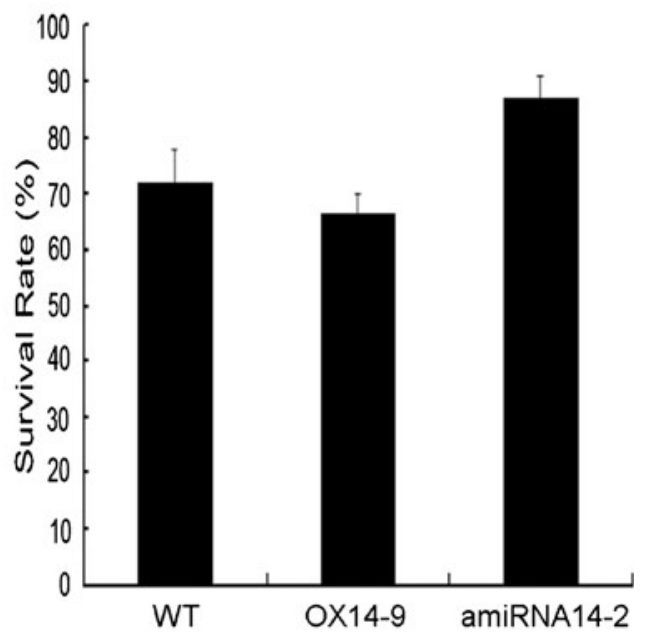

OX14-9
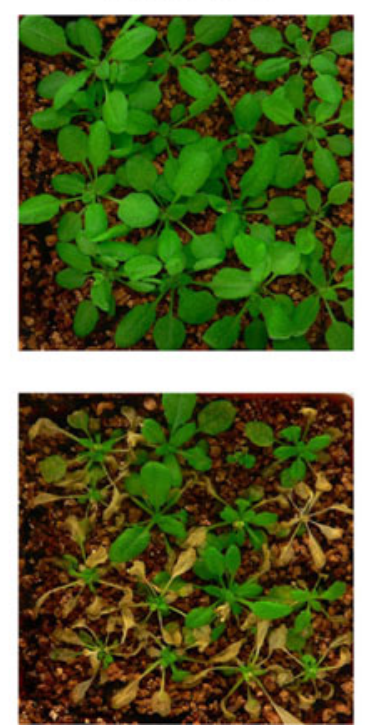

amiRNA14-2
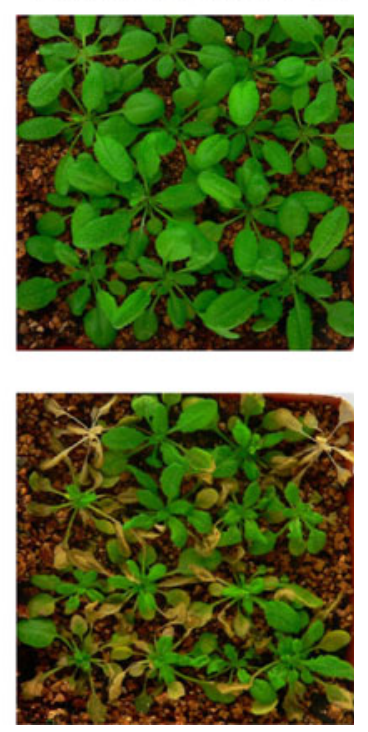

regulated in cold stress (Agarwal et al. 2006), whereas AtMYB 14 is down-regulated by cold treatment. It is logical that plants down-regulate negative regulators when facing cold stress and thus release expression of genes that protect against cold stress (Lee et al. 2001). It is still unknown why plants elevate the expression of AtMYB15 in low temperature since it is a negative regulator. In this study, we found that expression of $A t M Y B 15$ was not altered in either AtMYB14 overexpression lines or knock-down lines. This indicated that $A t M Y B 15$ expression is not affected by changes in expression of AtMYB14. Second, the downstream genes $R D 29 A$ and $C O R 15 A$ showed no changes in AtMYB 15 overexpression and knock-down lines (Lin and Thomashow 1992; Lee et al. 2001; Agarwal et al. 2006), whereas in AtMYB14 knock-down lines these genes are highly induced as expected. These differences suggest that the functional mechanisms of AtMYB14 and AtMYB15 in cold tolerance differ. The C-terminal 116-223 amino acids in AtMYB14 had low similarity to the corresponding region of AtMYB15. This region showed high transactivation activity (Fig. 3b). This may explain the molecular basis of the difference between AtMYB14 and AtMYB15.

The results of transactivation activity assays in yeast reveal that AtMYB14 may be a transcriptional activator, but the knock-down lines show increased tolerance to freezing by inducing expression of $C B F$ genes and their downstream genes in cold stress. This sounds conflicting, but is not unusual in plants. For example, WRKY48 has been 

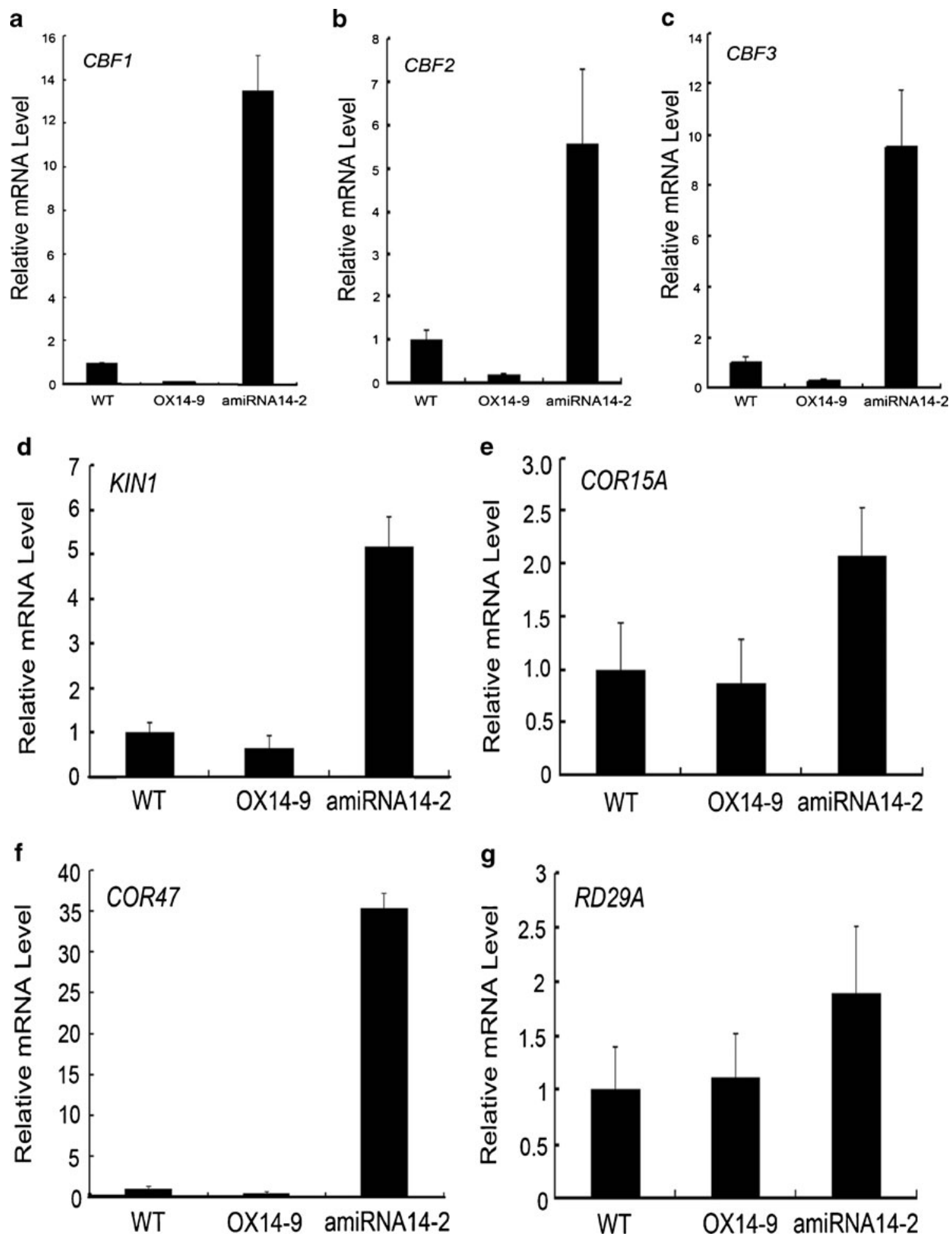

Fig. 6 Relative mRNA levels of cold responsive genes examined by real-time quantitative PCR. Expression levels of $C B F 1$ (a), $C B F 2$ (b), and $C B F 3$ (c) were increased to a much greater extent in amiRNA14-2 than in wild-type or OX14-9 lines under cold treatment. The expression levels of the downstream genes such as KINI (d), COR15A (e),

reported to have a powerful transcriptional activity, but is a negative regulator in plant basal defense (Xing et al. 2008). A possible reason for an activator functioning as a repressor

COR47 (f), and RD29A (g) in amiRNA14-2 lines were also elevated to a much higher level than those in wild-type or OX14-9 lines under low temperature. The gene expression level in wild type plants was set to 1.0. Error bars $\mathrm{SD}$ of biological replicates $(n=3)$

is that AtMYB14 may not regulate $C B F$ genes directly, but might activate other transcription factors that repress the expression of $C B F$ genes. Alternatively, AtMYB14 may 
interact with some repressor and recruit it to the promoter regions of target genes. Although the actual mechanisms by which AtMYB14 regulates cold tolerance by downregulating $C B F$ genes are still unknown, the data reported here suggest strongly that it is involved in the plant response to low temperature.

In summary, AtMYB14 is transcription activator that plays an important role in cold tolerance by affecting $C B F$ genes. This provides new information to increase our understanding of the complex network of transcriptional control in the plant response to cold stress.

Acknowledgments This work was supported by National Key Basic Research Program of People's Republic of China (9732011CB100101). We thank Professor Li-Jia Qu (Peking University, Beijing, China) for great comments on the manuscripts.

Open Access This article is distributed under the terms of the Creative Commons Attribution License which permits any use, distribution, and reproduction in any medium, provided the original author(s) and the source are credited.

\section{References}

Agarwal M, Hao Y, Kapoor A, Dong CH, Fujii H, Zheng X, Zhu JK (2006) A R2R3-type MYB transcription factor is involved in the cold regulation of CBF genes and in acquired freezing tolerance. $\mathrm{J}$ Biol Chem 281:27636-37645

Baker SS, Wilhelm KS, Thomashow MF (1994) The 5'-region of Arabidopsis thaliana cor15a has cis-acting elements that confer cold-, drought-, and ABA-regulated gene expression. Plant Mol Biol 24:701-713

Chen YH, Yang XY, He K, Liu MH, Li JG, Gao ZF, Lin ZQ, Zhang YF, Wang XX, Qiu XM, Shen YP, Zhang L, Deng XH, Luo JC, Deng XW, Chen ZL, Gu HY, Qu LJ (2006a) The MYB transcription factor superfamily of Arabidopsis: expression analysis and phylogenetic comparison with the rice MYB family. Plant Mol Biol 60:107-124

Chen YH, Zhang XB, Wei Wu, Chen ZL, Gu HY, Qu LJ (2006b) Overexpession of the wounding-responsive gene AtMYB15 activates the shikimate pathway in Arabidopsis. J Integr Plant Biol 48:1084-1095

Chinnusamy V, Ohta M, Kanrar S, Lee BH, Hong X, Agarwal M, Zhu JK (2003) ICE1: a regulator of cold-induced transcriptome and freezing tolerance in Arabidopsis. Genes Dev 17:1043-11054

Dong CH, Agarwal M, Zhang YY, Xie Q, Zhu JK (2006) The negative regulator of plant cold responses, HOS1, is a RING E3 ligase that mediates the ubiquitination and degradation of ICE1. Proc Natl Acad Sci USA 103:8281-8286

Gilmour SJ, Artus NN, Thomashow MF (1992) cDNA sequence analysis and expression of two cold-regulated genes of Arabidopsis thaliana. Plant Mol Biol 18:13-21

Gilmour SJ, Zarka DG, Stockinger EJ, Salazar MP, Houghton JM, Thomashow MF (1998) Low temperature regulation of the Arabidopsis CBF family of AP2 transcriptional activators as an early step in cold-induced COR gene expression. Plant J 16:433-442

Jiang FL, Wang F, Wu Z, Li Y, Shi GJ, Hu JD, Hou XL (2011) Components of the Arabidopsis CBF cold-response pathway are conserved in non-heading Chinese cabbage. Plant Mol Biol Rep 29:525-532
Kranz HD, Denekamp M, Greco R, Jin H, Leyva A, Meissner RC, Petroni K, Urzainqui A, Bevan M, Martin C, Smeekens S, Tonelli C, Paz-Ares J, Weisshaar B (1998) Towards functional characterization of the members of the R2R3-MYB gene family from Arabidopsis thaliana. Plant J 16:263-276

Kurkela S, Franck M (1990) Cloning and characterization of a cold- and ABA-inducible Arabidopsis gene. Plant Mol Biol 15:137-144

Lee H, Xiong LM, Gong ZZ, Ishitani M, Stevenson B, Zhu JK (2001) The Arabidopsis HOS1 gene negatively regulates cold signal transduction and encodes a RING finger protein that displays cold-regulated nucleo-cytoplasmic partitioning. Genes Dev 15:912-924

Li JG, Li XJ, Guo L, Lu F, Feng XJ, He K, Wei LP, Chen ZL, Qu LJ, Gu HY (2006a) A subgroup of MYB transcription factor genes undergoes highly conserved alternative splicing in Arabidopsis and rice. J Exp Bot 57:1263-1273

Li JG, Yang XY, Wang Y, Li XJ, Gao ZF, Pei M, Chen ZL, Qu LJ, Gu HY (2006b) Two groups of MYB transcription factors share a motif which enhances trans-activation activity. Biochem Biophys Res Commun 341:1155-1163

Lin C, Thomashow MF (1992) A cold-regulated Arabidopsis gene encodes a polypeptide having potent cryoprotective activity. Biochem Biophys Res Commun 183:1103-1108

Liu Q, Kasuga M, Sakuma Y, Abe H, Miura S, Yamaguchi-Shinozaki K, Shinozaki K (1998) Two transcription factors, DREB1 and DREB2, with an EREBP/AP2 DNA binding domain, separate two cellular signal transduction pathways in drought- and low temperature-responsive gene expression, respectively, in Arabidopsis. Plant Cell 10:1391-1406

Liu JJ, Zhang YY, Qin GJ, Tomohiko T, Norihiro S, Luo G, Sun KT, Shi DQ, Shiori A, Zheng NY, Takashi A, Atsuhiro O, Yang WC, Masaaki U, Xie Q, Gu HY, Qu LJ (2008) Targeted degradation of the cyclin-dependent kinase inhibitor ICK4/KRP6 by RING-type E3 ligases is essential for mitotic cell cycle progression during Arabidopsis gametogenesis. Plant Cell 20:1538-1554

Liu L, Cao XL, Bai R, Yao N, Li LB, He CF (2012) Isolation and characterization of the cold-induced phyllostachys edulis ap2/erf family transcription factor, peDREB1. Plant Mol Biol Rep 30:679-689

Nordin K, Vahala T, Palva ET (1993) Differential expression of two related, low-temperature-induced genes in Arabidopsis thaliana (L.) Heynh. Plant Mol Biol 21:641-653

Novillo F, Alonso JM, Ecker JR, Salinas J (2004) CBF2/DREB1C is a negative regulator of CBF1/DREB1B and CBF3/DREB1A expression and plays a central role in stress tolerance in Arabidopsis. Proc Natl Acad Sci USA 101:3985-3990

Novillo F, Medina J, Salinas J (2007) Arabidopsis CBF1 and CBF3 have a different function than $\mathrm{CBF} 2$ in cold acclimation and define different gene classes in the CBF regulon. Proc Natl Acad Sci USA 104:21002-21007

Qin GJ, Gu HY, Zhao YD, Ma ZQ, Shi GL, Yang Y, Eran P, Chen HD, Liu MH, Chen ZL, Qu LJ (2005) An indole-3-acetic acid carboxyl methyltransferase regulates Arabidopsis leaf development. Plant Cell 17:2693-2704

Riechmann JL, Ratcliffe OL (2000) A genomic perspective on plant transcription factors. Curr Opin Plant Biol 3:423-434

Schwab R, Ossowski S, Riester M, Warthmann N, Weigel D (2006) High specific gene silencing by artificial microRNAs in Arabidopsis. Plant Cell 18:1121-1133

Stockinger EJ, Gilmour SJ, Thomashow MF (1997) Arabidopsis thaliana $\mathrm{CBF} 1$ encodes an AP2 domain-containing transcription activator that binds to the $\mathrm{C}$ repeat/DRE, a cis-acting DNA regulatory element that stimulates transcription in response to low temperature and water deficit. Proc Natl Acad Sci USA 94:1035-1040

Stracke R, Werber M, Weisshaar B (2001) The R2R3-MYB gene family in Arabidopsis thaliana. Curr Opin Plant Biol 4:447-456 
Thomashow MF (1998) Role of cold-responsive genes in plant freezing tolerance. Plant Physiol 118:1-7

Thomashow MF (1999) Plant cold acclimation: freezing tolerance genes and regulatory mechanisms. Annu Rev Plant Physiol Plant Mol Biol 50:571-599

Viswanathan C, Zhu JK (2002) Molecular genetic analysis of coldregulated gene transcription. Philos Trans R Soc Lond B 357:877-886

Wang CT, Yang Q, Wang CT (2011) Isolation and functional characterization of ZmDBP2 encoding a dehydration-responsive elementbinding protein in Zea mays. Plant Mol Biol Rep 29:60-68

Xing SF, Qin GJ, Shi Y, Ma ZQ, Chen ZL, Gu HY, Qu LJ (2007) GAMT2 encodes a methyltransferase of gibberellic acid that is involved in seed maturation and germination in Arabidopsis. $\mathrm{J}$ Integr Plant Biol 49:368-381

Xing DH, Lai ZB, Zheng ZY, Vinod KM, Fan BF, Chen ZX (2008) Stressand pathogen-induced Arabidopsis WRKY48 is a transcriptional activator that represses plant basal defense. Mol Plant 1:459-470

Xiong L, Schumaker KS, Zhu JK (2002) Cell signaling during cold, drought and salt stresses. Plant Cell 14:S165-S183
Yamaguchi-Shinozaki K, Shinozaki K (1994) A novel cis-acting element in an Arabidopsis gene is involved in responsiveness to drought, low-temperature, or high-salt stress. Plant Cell 6:251264

Ye R, Yao QH, Xu ZH, Xue HW (2004) Development of an efficient method for the isolation of factors involved in gene transcription during rice embryo development. Plant J 38:348-357

Yi L, Qu LJ, Chang SR, Su YJ, Gu HY, Chen ZL (2002) Two nuclear localization signals required for the nuclear localization of rice ribosomal protein S4. Plant Sci 162:251-256

Zhang X, Guo XP, Lei CL, Cheng ZJ, Lin QB, Wang JL, Wu FQ, Wang J, Wan JM (2011) Overexpression of SlCZFP1, a novel TFIIIA-type zinc finger protein from Tomato, confers enhanced cold tolerance in transgenic Arabidopsis and Rice. Plant Mol Biol Rep 29:185-196

Zhu JH, Shi HZ, Byeong-ha L, Barbare D, Cheng S, Vicki S, Zhu JK, Paul MH, Ray AB (2004) An Arabidopsis homeodomain transcription factor gene, HOS9, mediates cold tolerance through a CBF-independent pathway. Proc Natl Acad Sci USA 101:98739878 